\title{
EFEITO DO CLORETO DE MEPIQUAT E DO THIDIAZURON SOBRE ALGUMAS CARACTERÍSTICAS DAS SEMENTES DO ALGODOEIRO1
}

\author{
FERNANDO MENDES LAMAS² e MANOELLUIZ FERREIRAATHAYDE ${ }^{3}$
}

\begin{abstract}
RESUMO - Com o objetivo de avaliar o efeito de diferentes doses de cloreto de mepiquat $(0,50,75,100$ e $125 \mathrm{~g} / \mathrm{ha})$ e de thidiazuron $(0,45,60$ e $75 \mathrm{~g} / \mathrm{ha}$ ) sobre as características fisiológicas de sementes de algodoeiro, foi conduzido, na UNESP, Jaboticabal, SP, um experimento inteiramente casualizado, num arranjo fatorial $(5 \times 4)$, com quatro repetições. Com o aumento da dose de cloreto de mepiquat, verificouse aumento do peso de 100 sementes e do índice de velocidade de emergência de plântulas. O efeito do cloreto de mepiquat e do thidiazuron sobre a matéria seca das plântulas foi antagônico. A porcentagem de germinação e o comprimento do hipocótilo não foram significativamente afetados pelo cloreto de mepiquat, pelo thidiazuron e pela interação entre eles.
\end{abstract}

Termos para indexação: Gossypium hirsutum, regulador de crescimento, desfolhante, germinação, vigor.

\section{EFFECT OF MEPIQUAT CHLORIDE AND THIDIAZURON ON SOME CHARACTERISTICS OF COTTON SEED}

\begin{abstract}
With the objective of evaluating the effect of different doses of mepiquat chloride $(0,50$, $75,100$ and $125 \mathrm{~g} / \mathrm{ha})$, and thidiazuron $(0,45,60$ and $75 \mathrm{~g} / \mathrm{ha})$ on the physiological characteristics of cotton seed, an experiment was carried out at UNESP, Jaboticabal, São Paulo State, Brazil. The experimental design was a completely randomized block design and the treatments were arranged in a $5 \times 4$ factorial replicated four times. The results showed that in increasing mepiquat chloride doses resulted in higher 100 seed weight and faster emergence indices. As for seedling dry matter, mepiquat chloride and thidiazuron showed antagonic effects. The germination percentage and the hypocotil length were not affected significantly by either chemical, alone or in combination.
\end{abstract}

Index terms: Gossypium hirsutum, growth regulator, defoliant, germination, vigour.

\section{INTRODUÇ̃̃O}

Os efeitos potenciais dos reguladores de crescimento, na cultura do algodoeiro (Gossypium hirsutum L.) são a redução do crescimento vegetativo, a melhora da arquitetura da planta, o aumento da retenção de frutos nas primeiras posições dos ramos frutíferos, o aumento da precocidade, e as melhoras na eficiência da colheita

\footnotetext{
${ }^{1}$ Aceito para publicação em 7 de maio de 1999.

${ }^{2}$ Eng. Agr., Dr., Embrapa-Centro de Pesquisa Agropecuária do Oeste (CPAO), Caixa Postal 661, CEP 79804-970 Dourados, MS. E-mail: lamas@cpao.embrapa.br

${ }^{3}$ Eng., Agr., Dr., Prof. Titular, Dep. de Fitotecnia, Faculdade de Ciências Agrárias e Veterinárias de Jaboticabal, UNESP, Rod. Carlos Tonani, Km 5, CEP 14870-000 Jaboticabal, SP.
}

e na qualidade do produto colhido (Barbosa \& Castro, 1983; Kerby et al., 1986; Reddy et al., 1990; McConnell et al., 1992).

Especialmente na colheita mecanizada, o uso de desfolhantes é uma prática indispensável. Bariola et al. (1990) e Busoli (1991) citam que a utilização de desfolhante, na cultura do algodoeiro, permite a antecipação da colheita, que, por sua vez, pode contribuir para reduzir o ataque de pragas tardias como a lagarta-rosada (Pectinophora gossypiella (Saunders, 1844)) e o bicudo-doalgodoeiro (Anthonomus grandis (Boheman, 1843)).

O potencial germinativo de uma semente é influenciado pelas condições a que foram submetidas durante a produção (Mecelis et al., 1991).

De acordo com Paolinelli (1986), as épocas de colheita do algodão, que permitem obter sementes de boa qualidade fisiológica, foram aquelas em que 
$40 \%$ a $70 \%$ dos capulhos se encontravam abertos. À medida que a colheita é retardada, existe a tendência de queda da qualidade das sementes. Com a utilização de desfolhantes, a colheita pode ser antecipada.

A literatura sobre os efeitos dos reguladores de crescimento e desfolhantes, nas características fisiológicas das sementes do algodoeiro, é escassa.

Objetivou-se avaliar os efeitos de diferentes doses de cloreto de mepiquat e de thidiazuron sobre o desempenho das sementes.

\section{MATERIAL E MÉTODOS}

As sementes utilizadas no presente trabalho foram produzidas em Ponta Porã, MS, no ano agrícola de 1994/95. As plantas que deram origem às sementes receberam aplicações de cloreto de mepiquat, cujas doses e esquema de parcelamento adotados se encontram na Tabela 1. Aos 136 dias após a emergência das plântulas, quando $70 \%$ dos capulhos estavam abertos, foram aplicadas as seguintes doses de thidiazuron: 0, 45, $60 \mathrm{e}$ $75 \mathrm{~g} / \mathrm{ha}$ de i.a.

As sementes da cultivar CNPA ITA 90 foram obtidas por meio da colheita de 30 capulhos localizados no terço médio das plantas, de acordo com a metodologia sugerida por Sabino et al. (1975). O línter não foi retirado.

Os testes fisiológicos foram realizados no Laboratório de Sementes do Departamento de Fitotecnia da Faculdade de Ciências Agrárias e Veterinárias de Jaboticabal, SP.

TABELA 1. Doses de cloreto de mepiquat, de acordo com o tratamento e com a época de aplicação, em número de dias após a emergência. Ponta Porã, MS, 1996.

\begin{tabular}{crrrr}
\hline \multirow{2}{*}{ Tratamento } & \multicolumn{2}{c}{ Época de aplicação (dias) } & \multirow{2}{*}{ Total } \\
\cline { 2 - 3 } & \multicolumn{1}{c}{42} & 60 & 73 & \\
\hline & ------1 & & \\
1 & 0,0 & 0,0 & 0,0 & 0,0 \\
2 & 12,5 & 12,5 & 25,5 & 50,0 \\
3 & 25,0 & 25,0 & 25,0 & 75,0 \\
4 & 0,0 & 50,0 & 50,0 & 100,0 \\
5 & 12,5 & 62,5 & 50,0 & 125,0 \\
\hline
\end{tabular}

Pesq. agropec. bras., Brasília, v.34, n.11, p.2015-2019, nov. 1999
O teste de germinação, conduzido em caixas com areia, seguiu as prescrições das Regras de Análise de Sementes (Brasil, 1992). A velocidade de emergência de plântulas foi determinada pela contagem diária das plântulas normais com hipocótilo de comprimento superior a $1 \mathrm{~cm}$, durante o período compreendido entre o quarto e o décimo segundo dia após a semeadura. Determinou-se o índice de velocidade de emergência das plântulas de acordo com Popinigis (1977).

No décimo segundo dia após a semeadura, foi medido o hipocótilo (mm) de 10 plântulas normais por repetição; em seguida, as plântulas foram acondicionadas em sacos de papel e levadas à estufa de circulação de ar forçada $65^{\circ} \mathrm{C}$ até a obtenção de peso constante, para a estimativa do peso da matéria seca $(\mathrm{mg})$.

O delineamento experimental utilizado foi inteiramente casualizado, num arranjo fatorial $5 \times 4$, com quatro repetições. Os tratamentos foram constituídos pela combinação dos níveis dos fatores cloreto de mepiquat e thidiazuron nas dosagens de $0,50,75,100$ e $125 \mathrm{~g} /$ ha e 0 , $45,60,75 \mathrm{~g} / \mathrm{ha}$, respectivamente. Os resultados obtidos foram submetidos à análise de variância, e posteriormente foi efetuada análise de regressão polinomial, de acordo com Banzatto \& Kronka (1992). Os dados de porcentagem de germinação foram transformados em arc sen $\sqrt{\mathrm{x} / 100}$.

\section{RESULTADOS E DISCUSSÃO}

O resumo da análise de variância dos dados referentes ao peso de 100 sementes, porcentagem de germinação, comprimento do hipocótilo, peso da matéria seca de plântulas e índice de velocidade de emergência encontram-se na Tabela 2.

$\mathrm{O}$ efeito do cloreto de mepiquat foi significativo $(\mathrm{P}<0,01)$ no tocante à variável peso de 100 sementes, com os dados se ajustando a uma equação de segundo grau, com ponto de máximo na dose de 90 g/ha (Fig. 1). Em trabalhos desenvolvidos por Cia et al. (1984) e Carvalho et al. (1994a), foram encontrados resultados semelhantes. Cruz et al. (1982) não encontraram efeito significativo do cloreto de mepiquat sobre o peso de 100 sementes. No presente trabalho, foram estudadas doses maiores do que as estudadas pelos autores citados. $\mathrm{O}$ aumento do peso de 100 sementes, no que diz respeito ao algodoeiro, não é interessante, pois a correlação entre peso de 100 sementes e porcentagem de fibra é negativa (Carvalho et al., 1994b). Em trabalho desenvolvido por Paolinelli (1986), não se verifica correla- 
TABELA2. Resumo das análises de variância (quadrados médios) dos dados de peso de 100 sementes $\left(\mathrm{P}_{100}\right)$, porcentagem de germinação (G), peso da matéria seca de 10 plântulas (MS), comprimento do hipocótilo (CH) e índice de velocidade de emergência (IVE). Ponta Porã, MS, 1996.

\begin{tabular}{lcccccc}
\hline \multirow{2}{*}{ Fonte de variação } & \multirow{2}{*}{ G.L } & \multicolumn{5}{c}{ Quadrado médio } \\
\cline { 3 - 7 } & & $\mathrm{P}_{100}$ & $\mathrm{G}$ & $\mathrm{MS}$ & $\mathrm{CH}$ & ÍVE \\
\hline Cloreto de mepiquat (CM) & 4 & $1,74^{* *}$ & $124,80^{\mathrm{ns}}$ & $126,98^{*}$ & $1,18^{\mathrm{ns}}$ & $2,48^{\mathrm{ns}}$ \\
Thidiazuron (TDZ) & 3 & $0,05^{\mathrm{ns}}$ & $3,73^{\mathrm{ns}}$ & $0,54^{*}$ & $0,24^{\mathrm{ns}}$ & $0,29^{\mathrm{ns}}$ \\
CM x TDZ & 12 & $0,13^{\mathrm{ns}}$ & $36,40^{\mathrm{ns}}$ & $60,39^{\mathrm{ns}}$ & $0,34^{\mathrm{ns}}$ & $0,35^{\mathrm{ns}}$ \\
Resíduo & 60 & 0,24 & 51,25 & 44,78 & 0,54 & 0,99 \\
\hline C.V. $(\%)$ & & 2,70 & 8,32 & 11,88 & 6,34 & 9,74 \\
\hline
\end{tabular}

ns,${ }^{*} \mathrm{e}^{* *}$ Não-significativo e significativo a $5 \%$ e $1 \%$ de probalidade pelo teste $\mathrm{F}$, respectivamente

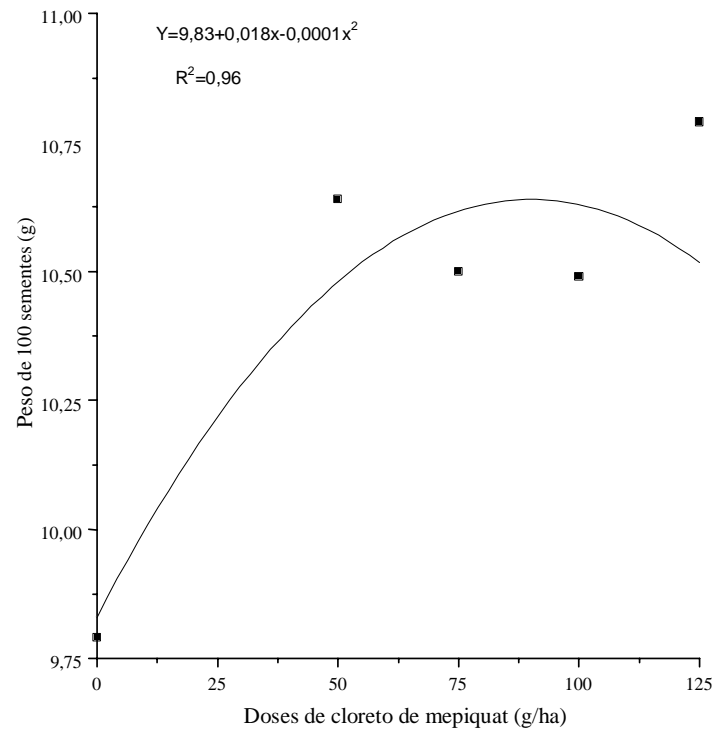

FIG. 1. Efeito de doses de cloreto de mepiquat sobre o peso de 100 sementes de algodão. Ponta Porã, MS, 1996.

ção significativa entre peso de 100 sementes e desempenho fisiológico. Os efeitos do thidiazuron e da interação entre cloreto de mepiquat e thidiazuron não foram significativos $(\mathrm{P}>0,05)$; resultados semelhantes foram obtidos por Cruz et al. (1982) ao estudarem o efeito do thidiazuron.

A porcentagem de germinação não foi significativamente influenciada pelo cloreto de mepiquat, pelo thidiazuron, bem como pela interação entre ambos. Esses resultados corroboram os obtidos por Cruz et al. (1982) e Rios \& Martinez (1983).
Os efeitos do cloreto de mepiquat e do thidiazuron sobre o peso da matéria seca de plântulas foram significativos (Tabela 2). Com o aumento da dose de cloreto de mepiquat, foi verificado aumento linear do peso da matéria seca das plântulas (Fig. 2). O cloreto de mepiquat aumenta a eficiência fotossintética e o metabolismo do nitrogênio do algodoeiro (Athayde, 1980; Hodges et al.,1991) o que, embora não tenha sido objeto de avaliação no presente trabalho, constitui uma hipótese para explicação dos resultados obtidos, ante a possibilidade de o produto aumentar o peso de 100 sementes e, em decorrência, promover o aumento do peso da matéria seca das plântulas. Com o aumento da dose de thidiazuron, verificou-se redução linear do peso da matéria seca das plântulas (Fig. 3). Considerando que as sementes utilizadas neste estudo são oriundas de frutos localizados no terço médio das plantas, não se encontra uma explicação lógica para esse efeito, pois quando da aplicação do produto, essas sementes já estavam fisiologicamente maduras.

O comprimento do hipocótilo e o índice de velocidade de emergência não foram significativamente influenciados $(\mathrm{P}>0,05)$ pelos tratamentos (Tabela 2); entretanto, quando se procedeu ao desdobramento dos graus de liberdade do fator cloreto de mepiquat, o efeito foi linear (Fig. 4). Tais resultados evidenciam que o vigor das sementes, quando considerado o índice de velocidade de emergência, cresceu com o aumento da dose de cloreto de mepiquat. 


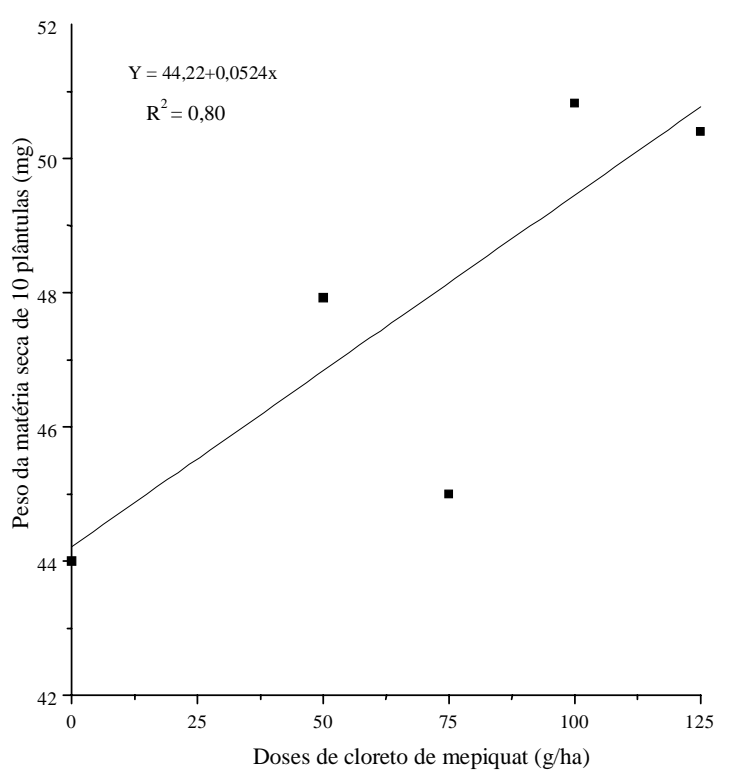

FIG. 2. Efeito de doses do cloreto de mepiquat sobre a matéria seca de plântulas de algodão. Ponta Porã, MS, 1996.

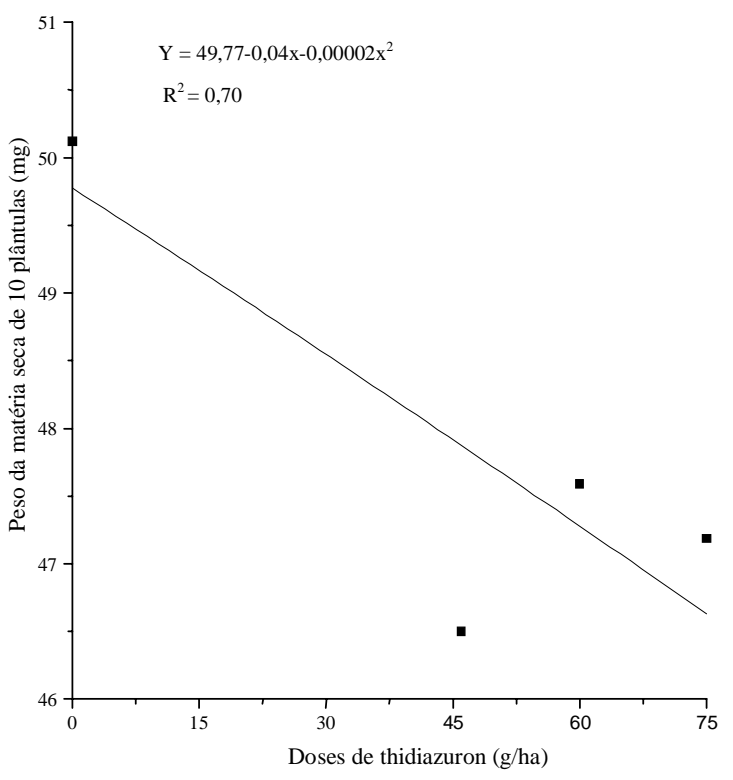

FIG. 3 . Efeito de doses de thidiazuron sobre o peso da matéria seca de plântulas de algodão. Ponta Porã, MS, 1996.

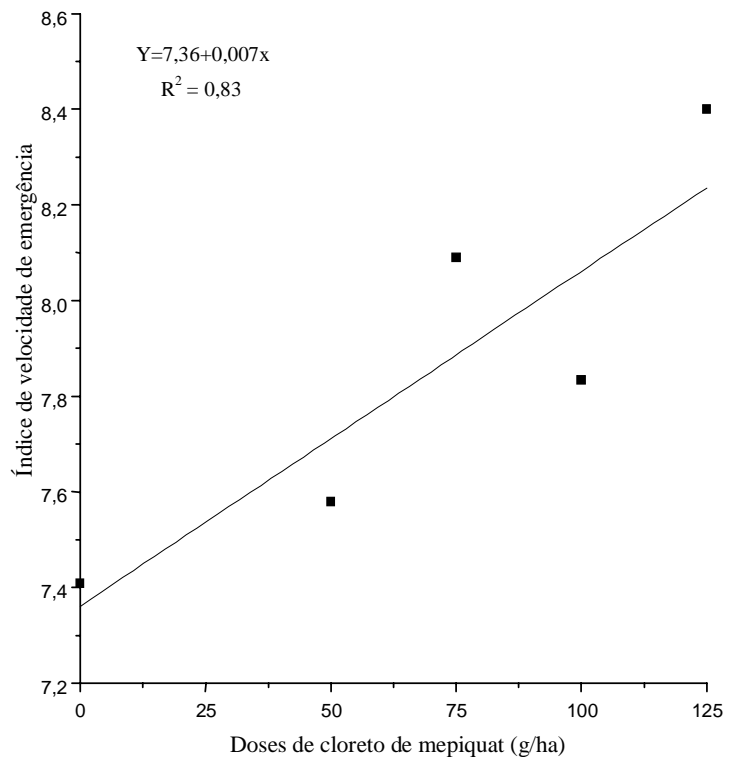

FIG. 4 . Efeito de doses de cloreto de mepiquat sobre o índice de velocidade de emergência de sementes de algodão. Ponta Porã, MS, 1996.

\section{CONCLUSÕES}

1. O maior peso de 100 sementes é obtido com a dose de $90 \mathrm{~g} / \mathrm{ha}$ de cloreto de mepiquat.

2. O índice de velocidade de emergência de plântulas aumenta linearmente com a elevação da dose de cloreto de mepiquat.

3. O peso da matéria seca das plântulas cresce com o aumento da dose de cloreto de mepiquat $\mathrm{e}$ decresce com o aumento da dose de thidiazuron.

4. As doses de cloreto de mepiquat e de thidiazuron estudadas não interferem na porcentagem de germinação e no comprimento do hipocótilo.

\section{REFERÊNCIAS}

ATHAYDE, M.L.F. Efeitos do $\mathbf{N}$ de cloreto de clorocolina (CCC) no metabolismo nitrogenado e em algumas características do algodoeiro (Gossypium hirsutum L.). Piracicaba: ESALQ, 1980. 94p. Tese de Doutorado. 
BANZATTO, D.A.; KRONKA, S.N. Experimentação agrícola. 2.ed. Jaboticabal: FUNEP, 1992. 247p

BARBOSA, L.M.; CASTRO, P.R.C. Desenvolvimento e produtividade de algodoeiros sob efeito de reguladores vegetais. Anais da Escola Superior de Agricultura "Luiz de Queiroz", Piracicaba, v.40, n.1, p.33-86, 1983.

BARIOLA, L.A.; CHU, C.C.; HENNEBERRY, T.J. Timing applications of plant growth regulators and last irrigation for pink bollworm (Lepidoptera Gelechiidae) control. Journal of Economic Entomology, Lanham, v.83, n.3, p.1074-1079, 1990.

BRASIL. Ministério da Agricultura e Reforma Agrária. Regras para análise de sementes. Brasília: DNDV/CLAV, 1992.365p

BUSOLI, A.C. Práticas culturais, reguladores de crescimento, controle químico e feromônios no manejo integrado de pragas do algodoeiro In: DEGRANDE, P.E. (Ed.). Bicudo do algodoeiro: manejo integrado de pragas. Dourados: UFMS/Embrapa-UEPAE de DOURADOS, 1991. p.29-52

CARVALHO, L.H.; CHIAVEGATO, E.J.; CIA, E.; KONDO, J.I.; SABINO, J.C.; PETTINELLI JÚNIOR, A.; BORTOLETTO, N.; GALLO, P.B. Fitorreguladores de crescimento e capação na cultura algodoeira. Bragantia, Campinas, v.53, n.2, p.247-254, 1994a

CARVALHO, L.P.; CRUZ, C.D.; MORAES, C.F. de Correlações genotípicas, fenotípicas e ambientais em algodoeiro (Gossypium hirsutum L. var. latifolium Hutch.). Revista Ceres, Viçosa, v.41, n.236, p.407-418, 1994b.

CIA, E.; CARVALHO, L.H.; KONDO, J.I.; FUZATTO, M.G; BORTOLETTO, N.; GALLO, P.B.; CRUZ, L.S.P.; SABINO, N.P.; PETINELLI JÚNIOR, A.; MARTINS, A.L.M; SILVEIRA, J.C.O. Efeito do cloreto de clorocolina e cloreto de mepiquat na cultura do algodão (Gossypium hirsutum L.). Planta Daninha, Campinas, v.7, n.2, p. 23-36, 1984.
CRUZ, L.S.P.; SABINO, N.P.L.; TOLEDO, N.M.P Efeitos do cloreto de mepiquat empregado como fitorregulador sobre algodoeiro herbáceo (Gossypium hirsutum L.). Planta Daninha, Campinas, v.5, n.1, p.15-22, 1982

HODGES, H.F.; REDDY, V.R.; REDDY, K.R. Mepiquat chloride and temperature effects on photosynthesis and respiration o fruiting cotton. Crop Science, Madison, v.31, n.5, p.1302-1308, 1991.

KERBY, T.A.; HAKE, K.; KEELEY, M. Cotton fruiting modification with mepiquat chloride. Agronomy Journal, Madison, v.78, n.5, p.907-912, 1986.

McCONNELL, J.S.; BAKER, W.H.; FRIZZELL, B.S.; VARVIL, J.J. Response of cotton to nitrogen fertilization and early multiple applications of mepiquat chloride. Journal of Plant Nutrition, Monticello, v.15, n.4, p.457-468, 1992.

MECELIS, N.R.; SCHAMMASS, E.A.; DIAS, L.M.G.S. Efeitos da adubação nitrogenada sobre a germinação de sementes de capim ramirez. Revista Brasileira de Sementes, Brasília, v.13, n.1, p.53-57, 1991

PAOLINELLI, G.P. Influência de três épocas de colheita sobre a qualidade fisiológica de sementes de algodão em Minas Gerais. Revista Brasileira de Sementes, Brasília, v.8, n.2, p.77-83, 1986.

POPINIGIS, F. Fisiologia da semente. Brasília: AGIPLAN, 1977. 289p.

REDDY, V.R.; BAKER, D.N.; HODGES, H.F. Temperature and mepiquat chloride on cotton canopy architecture. Agronomy Journal, Madison, v.82, n.2, p.190-195, 1990.

RIOS, PC.; MARTINEZ, R.P. Efecto del cloruro de mepiquat sobre la planta del algodonero, Gossypium hirsutum L. Agricultura Técnica en México, México, v.9, n.1, p.35-44, 1983.

SABINO, N.P.; LAZARINI, J.F.; GRIDI-PAPP, I.L.; FUZATTO, M.G.; GROSSI, J.M.M. Estudo de amostragens de capulhos em canteiros experimentais de algodão. Bragantia, Campinas, v.34, n.8, p.6369,1975 